\title{
Atherogenic Indices as Markers for Risk of Myocardial Infarction in Obese
}

Authors

\section{Ayesha Jabeen ${ }^{1}$, Gulam Saidunnisa Begum*2, J. Rama Rao ${ }^{3}$}

${ }^{1}$ Assistant Professor, Department of Biochemistry, Mahavir Institute of Medical sciences, Vikarabad,

Telangana State, India

Email:ayesha2k3@gmail.com

${ }^{2}$ Associate Professor, Department of Biochemistry, Mahavir Institute of Medical sciences, Vikarabad, Telangana State, India

${ }^{3}$ Professor, Department of Biochemistry, Malla Reddy Medical College for Women, RR dist, Telangana

State India

Corresponding Author

\section{Gulam Saidunnisa Begum}

Associate Professor, Department of Biochemistry, Mahavir Institute of Medical sciences, Vikarabad,

Telangana State, India

Email: gulambegum1967@gmil.com

\begin{abstract}
The rising prevalence of obesity in India has a direct correlation with the increasing prevalence of obesity-related co-morbidities; hypertension, the metabolic syndrome, dyslipidemia, type 2 diabetes mellitus (T2DM), and cardiovascular disease (CVD).

Methods: A case control study was conducted in the department of biochemistry, Osmania general hospital, Hyderabad with the objective of assessing the discriminatory power of atherogenic indices over individual lipids in risk assessment of Myocardial infarction in obese. Cases were categorized into obese and non obese MI group depending on the BMI. Control groups were selected from the outpatient department of Osmania General Hospital. Control groups were classified into healthy controls and obese controls basing on BMI.

Results: The mean values of BMI , WHR , TC, TAG, LDL-C,CRR, AC, AIP are higher in obese controls when compared to healthy controls, HDL-C is lower in obese control group when compared to healthy control group. The BMI exhibited better discriminatory power than WHR. AIP and TAG exhibited the highest combined sensitivity and specificity followed by AC and CRR, TC and LDL-C in discriminating healthy controls and obese controls.

Conclusion: We conclude the present study showed atherogenic indices were found to be better markers in explaining the pathogenesis of atherosclerosis. Among them AIP had the highest sensitivity and specificity followed by cardiac risk ratio, atherogenic coefficient and TAG in predicting the future risk of development of atherosclerosis in obese.

Keywords: Cardiac risk ratio (CRR), atherogenic coefficient $(A C)$ and atherogenic index of plasma (AIP). Hypertension, metabolic syndrome, dyslipidemia, type 2 diabetes mellitus (T2DM), and cardiovascular disease (CVD).
\end{abstract}




\section{Introduction}

Obesity is defined as an excess accumulation of fat in the body resulting in adverse effects on health of the individual. ${ }^{1}$ The prevalence of obesity is rising to epidemic proportions at an alarming rate in both developed and less developed countries around the world. ${ }^{2}$

In India, obesity is emerging as an important health problem particularly in urban areas, paradoxically co-existing with under nutrition. Almost 30-65\% of adult urban Indians are either overweight or obese or have abdominal obesity. The rising prevalence of obesity in India has a direct correlation with the increasing prevalence of obesity-related co-morbidities; hypertension, the metabolic syndrome, dyslipidemia, type 2 diabetes mellitus (T2DM), and cardiovascular disease (CVD). ${ }^{1}$

Obesity is characterized by a series of lipid disturbances, such as hypercholesterolemia, high fasting (and postprandial) triacylglycerol levels, low HDL cholesterol, high apolipoprotein B, high small dense lipoprotein particles and alterations of serum and tissue lipoprotein lipase (LPL) activity. $^{4}$

Dyslipidemia is known to increase platelets aggregation, fibrinogen levels and platelets activation inhibitor. In addition, an elevated total cholesterol (TC), triacylglycerols (TAG), lowdensity lipoprotein-cholesterol (LDL-C) and lowered high-density lipoprotein cholesterol (HDL-C) are conventional risk factors for myocardial infarction as well as the major cause of atherosclerosis. ${ }^{6}$

Myocardial infarction is the leading cause of death worldwide. According to World Health Organization (WHO), in 2002 nearly 7.2 million deaths resulted from coronary heart disease. Risk factors associated with myocardial infarction are found to be old age, smoking, high risk diet, excess alcohol, abdominal obesity, hypertension, diabetes mellitus and dyslipidemia. ${ }^{7}$ Obesity has been correlated to increased morbidity and mortality risk in various populations. Three simple measures of obesity are widely used in clinical practice; BMI (body mass index), WC (waist circumference) and waist-to-hip circumference ratio (WHR). The combined use of these may be better in identifying people at risk of CVD than either of them alone. ${ }^{8}$

High plasma concentrations of triacylglycerols is an independent and synergistic risk factor for cardiovascular diseases and is often found in hypertension, abnormal lipoprotein metabolism, obesity, insulin resistance and diabetes mellitus. Similarly high plasma concentrations of LDL and VLDL cholesterol is also a risk factor for cardiovascular disease and is often found in diabetes mellitus, hypertension and obesity. Another major and well-established risk factor for the development of cardiovascular diseases is decreased plasma concentrations of HDL cholesterol which often accompanies diabetes mellitus, hypertension, and obesity. ${ }^{10}$

Several lipoprotein ratios or atherogenic indices have been defined in an order to increase the predictive capacity of the lipid profile. These ratios can provide information on risk factors difficult to quantify by routine analysis and are better indicators of the metabolic and clinical interactions between various lipid fractions. ${ }^{11}$

Atherogenic indices are powerful indicators of the risk of heart disease, the higher the value, the higher the risk of developing cardiovascular disease and vice versa. Low atherogenic indices are protective against coronary heart disease. ${ }^{10}$ The three atherogenic indices include, cardiac risk ratio (CRR), atherogenic coefficient (AC), atherogenic index of plasma (AIP).

\section{Objectives}

The objective of the study is to assess the discriminatory power of atherogenic indices over individual lipids in risk assessment of Myocardial infarction in obese.

\section{Methods and Materials Setting}

A case control study was conducted in the department of biochemistry, Osmania general 
hospital, Hyderabad, after obtaining institutional ethical approval.

\section{Sources of Samples and Data}

Cases were selected from the admitted patients in the Department of cardiology, Osmania General Hospital. The clinical diagnosis of Myocardial infarction was based on the presence of classical changes of Myocardial Infarction along with characteristic ST changes in ECG and the rise/fall of cardiac markers. Cases were categorized into obese and non obese MI group depending on the BMI. Control groups were selected from the outpatient department of Osmania General Hospital. Control groups were classified into healthy controls and obese controls basing on BMI. Written informed consent was taken from cases and controls. Samples were analyzed for various parameters at the Department of Biochemistry, Osmania General Hospital

\section{Inclusion Criteria}

1. Healthy control group: Consists of 30 healthy controls with BMI 18.5 - 24.99 of age group 42$60 \mathrm{yrs}$.

2. Obese control group: Consists of 30 healthy obese controls with $\mathrm{BMI} \geq 30$ of age group 40-55 yrs.

3. Non Obese myocardial infarction patients: Consists of 30 non-obese patients with BMI $\leq 30$ admitted in the cardiology department of age group 40 - 80 yrs diagnosed with myocardial infarction within 48 hours of onset of symptoms. The classification of obese, healthy groups was according to W.H.O classification.

4. Obese myocardial infarction patients: Consists of 30 obese patients with $\mathrm{BMI} \geq 30$ admitted in the cardiology department of age group 40-70 yrs diagnosed with myocardial infarction within 48 hours of symptoms.

\section{Exclusion Criteria}

Diabetes Mellitus, Hypothyroidism, Cushings syndrome, chronic systemic illness, Hepatic impairment, renal disorder, Endocrine disorder and subjects on lipid lowering drugs, thiazide diuretics were excluded from the study.

\section{Specimen collection}

Overnight fasting blood samples of $5 \mathrm{ml}$ of was taken from control groups and cases groups by venipuncture in plain serum tube. Serum was separated within 1 hour and grossly hemolysed samples were excluded. The following parameters were analyzed:

1. Body mass index (BMI): was calculated by dividing weight (in kilograms) by height (in meters squared) for the individuals ${ }^{3}$. Controls were categorized depending on the BMI as healthy controls, obese controls with BMI 18.5 - 24.99, $\geq 30$ respectively. Similarly MI cases were categorized as non obese MI cases and obese MI cases depending on BMI 18.5 - 24.99, $\geq 30$ respectively.

2. WHR: Waist circumference was measured using a measuring tape at the approximate midpoint between the lower margin of the last palpable rib and the top of the iliac crest, hip circumference measurement was taken around the widest portion of the buttocks. Waist -hip ratio was calculated by dividing waist circumference by the hip circumference. $^{3}$ Waist-hip ratios cut off values are: $0.90 \mathrm{~cm}$ (Males) $0.85 \mathrm{~cm}$ (Females) Values above these are associated with increased metabolic risk and other health complications.

3. Serum Total Cholesterol ${ }^{19}$ was estimated with Cholesterol oxidase and peroxidase method (CHOD-PAP). Reference Range for TC*: Serum Total cholesterol TC $(\mathrm{mg} / \mathrm{dl})$, Desirable<200, Borderline high risk, $200-239$, High risk $>240$

4. Serum HDL-Cholesterol ${ }^{20}$ was estimated with PEG - CHOD - PAP, both are End point Assays. Reference Range for HDL-C* Serum HDL cholesterol HDL-C (mg / dl): Low risk $>60$, High risk $<40$. *Reference values are recommended by the US National Education Program Expert Panel (NCEP ATP III ) 
5. Serum Triacylglycerol ${ }^{21}$ was estimated with GPO-PAP; an End point assay. Reference ranges* for serum triacylglycerol levels (mg/dl), Normal Less than 150, Borderline high150 to 199 , High200 to 499, Very high $>500$.

6. Serum Ldl-Cholesterol is calculated indirectly using Friedwalds Equation: LDL Cholesterol $=$ Total Cholesterol - HDL Cholesterol - Triacylglycerol /5. Reference values for LDL-C ( mg/dl)*: Optimal<100, Near optimal:100-129, Borderline High:130-159, High:160-189, Very High $\geq 190{ }^{*} \quad$ Reference values are recommended by the US National Education Program Expert Panel (NCEP -ATP III )

Atherogenic Indices: ${ }^{22}$ are:

7. Cardiac Risk Ratio (CRR): It is calculated by dividing total cholesterol by HDL. CRR=Total cholesterol/HDL. Cardio vascular Risk stratification using CRR, Interpretation: High risk (3X): Ratio in males: 9.7-23.4, Ratio in females:7.2-11.0, Above average risk (2X): Ratio in males: 5.1-9.6, Ratio in females: 4.5-7.1, Average risk: Ratio in males: 3.5-5.0, Ratio in females: 3.4-4.4, Below average risk (1/2): Ratio in males: 1.0-3.4, Ratio in females: 1.0-3.3.

8. Atherogenic Coefficient (AC) this is calculated by using the formula

$\mathrm{AC}=($ Total Cholesterol $-\mathrm{HDL}-\mathrm{C}) / \mathrm{HDL}-\mathrm{C}$

Or $\quad \mathrm{AC}=$ Non HDL-C / HDL-C.
9. Atherogenic Index of Plasma this is calculated by using the formula

AIP $=\log$ (Triacyglycerol $/ \mathrm{HDL}$ ), Reference value for AIP: low Cardiovascular risk $<0.1$, medium Cardiovascular risk:0.1-0.24, high Cardiovascular risk: $>0.24$. For calculating AIP individual lipid values were converted to $\mathrm{mmol} / \mathrm{l}$ by using formula: Triacylglycerol $(\mathrm{mmol} / \mathrm{l})=$ triacyglycerol $(\mathrm{mg} / \mathrm{dl}) / 89$ and Cholesterol $(\mathrm{mmol} / \mathrm{l})=$ Cholesterol $(\mathrm{mg} / \mathrm{dl}) /$ 39

\section{Results}

The mean values of all parameters studied are higher in total cases studied when compared to total controls except HDL-C which are lower in cases compared to controls. The mean values of BMI, WHR, TC, TAG, LDL-C ,CRR, AC, AIP are higher in obese controls when compared to healthy controls, HDL-C is lower in obese control group when compared to healthy control group. The data was analyzed using SPSS software version 17.0. Descriptive results are expressed as mean and SD of various parameters in different groups.

The results were expressed in milligrams /deciliter for Serum Total Cholesterol, Serum HDL Cholesterol, Serum Triacylgerol, Serum LDL Cholesterol BMI expressed in $\mathrm{kg} / \mathrm{m}^{2}$ WHR, Cardiac Risk Ratio, Atherogenic coefficient, Atherogenic index of plasma have no units as they are ratios.

Table 1. Mean \pm S.D of studied parameters in all groups

\begin{tabular}{|l|c|c|c|c|c|c|c|c|}
\hline Parameter & \multicolumn{2}{|c|}{ Healthy controls } & \multicolumn{2}{|c|}{ Obese controls } & \multicolumn{2}{|c|}{ Non obese MI } & \multicolumn{2}{c|}{ Obese MI } \\
\hline & Mean & \pm S.D & Mean & \pm S.D & Mean & \pm S.D & Mean & \pm S.D \\
\hline BMI & 23.31 & 1.27 & 33.86 & 3.32 & 22.91 & 1.15 & 33.66 & 2.64 \\
\hline WHR & 0.81 & 0.05 & 1.08 & 0.24 & 0.80 & 0.05 & 1.06 & 0.13 \\
\hline TC & 141.83 & 18.9 & 205.1 & 43.4 & 187.66 & 37.1 & 221.2 & 47.71 \\
\hline HDL-C & 47.2 & 7.49 & 37 & 7.02 & 38.0 & 9.86 & 33.57 & 7.09 \\
\hline TAG & 108.96 & 28.0 & 191.3 & 45.3 & 146.16 & 43.5 & 200.16 & 37.88 \\
\hline LDL-C & 73.17 & 19.1 & 129.5 & 45.2 & 120.4 & 34.8 & 147.56 & 48.74 \\
\hline CRR & 3.04 & 0.62 & 5.65 & 1.13 & 5.17 & 1.42 & 6.86 & 2.14 \\
\hline AC & 2.04 & 0.62 & 4.65 & 1.13 & 4.17 & 1.42 & 5.86 & 2.14 \\
\hline AIP & 0.01 & 0.14 & 0.35 & 0.15 & 0.22 & 0.20 & 0.42 & 0.11 \\
\hline
\end{tabular}

In order to assess the significance of the differences observed in the mean values of different parameters observed in different groups studied, the data is subjected to ANOVA test. The

significance of difference is represented by $\mathrm{p}$ values and $\mathrm{p}$ value $<0.05$ is considered as significant. 
Table 2. Anova F value and P VALUE between cases and controls

\begin{tabular}{|l|c|c|}
\hline Parameter & F value & Significance P value \\
\hline BMI & 29.836 & $<0.001$ \\
\hline WHR & 34.958 & $<0.001$ \\
\hline TC & 18.190 & $<0.001$ \\
\hline HDL $-C$ & 14.011 & $<0.001$ \\
\hline TAG & 26.556 & $<0.001$ \\
\hline LDL-C & 15.524 & $<0.001$ \\
\hline CRR & 30.805 & $<0.001$ \\
\hline AC & 30.805 & $<0.001$ \\
\hline AIP & 34.064 & $<0.001$ \\
\hline
\end{tabular}

Figure. 1 Graphical representation of Mean \pm Figure 2 Graphical representation of Mean \pm SD $\mathrm{SD}$ of BMI in healthy controls, obese controls ,non obese MI cases and obese MI cases of WHR in healthy controls, obese controls, non obese MI cases and obese MI cases
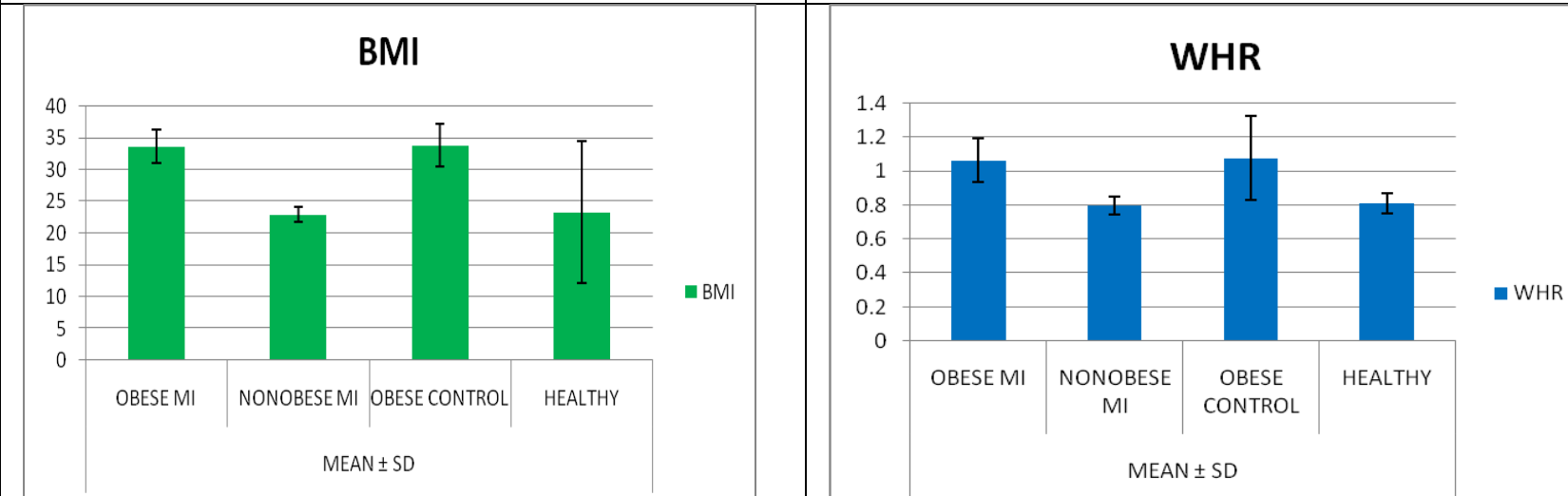

Figure 3. Graphical representation of TC, Figure 4 Graphical representation of CRR, HDL-C, TAG, LDL-C in healthy controls, Atherogenic coefficient in healthy controls, obese obese controls, non obese MI cases and obese controls, non obese MI cases and obese MI cases MI cases
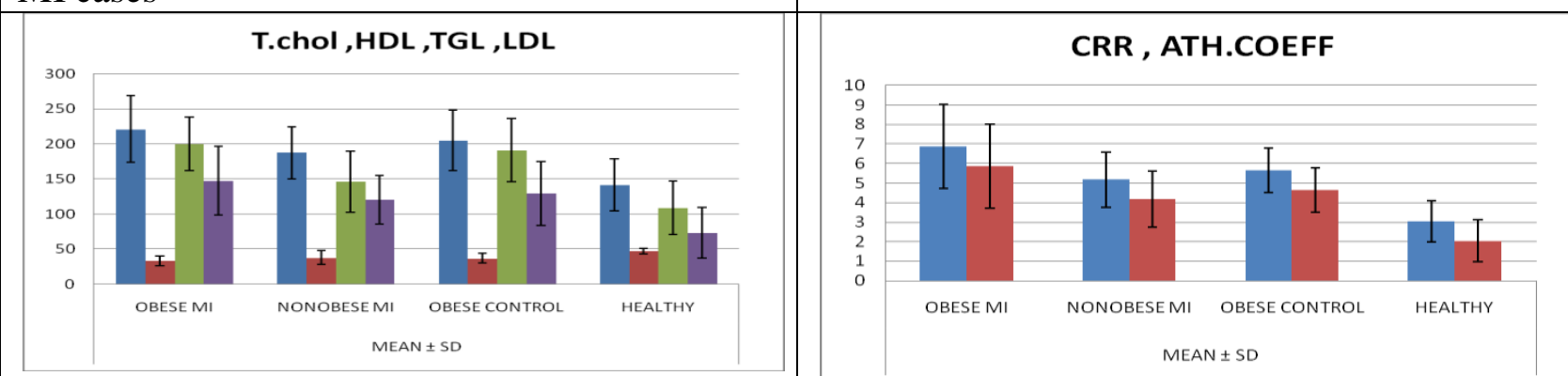

Figure 5 Graphical representation of AIP in healthy controls, obese controls ,non obese cases and obese MI cases

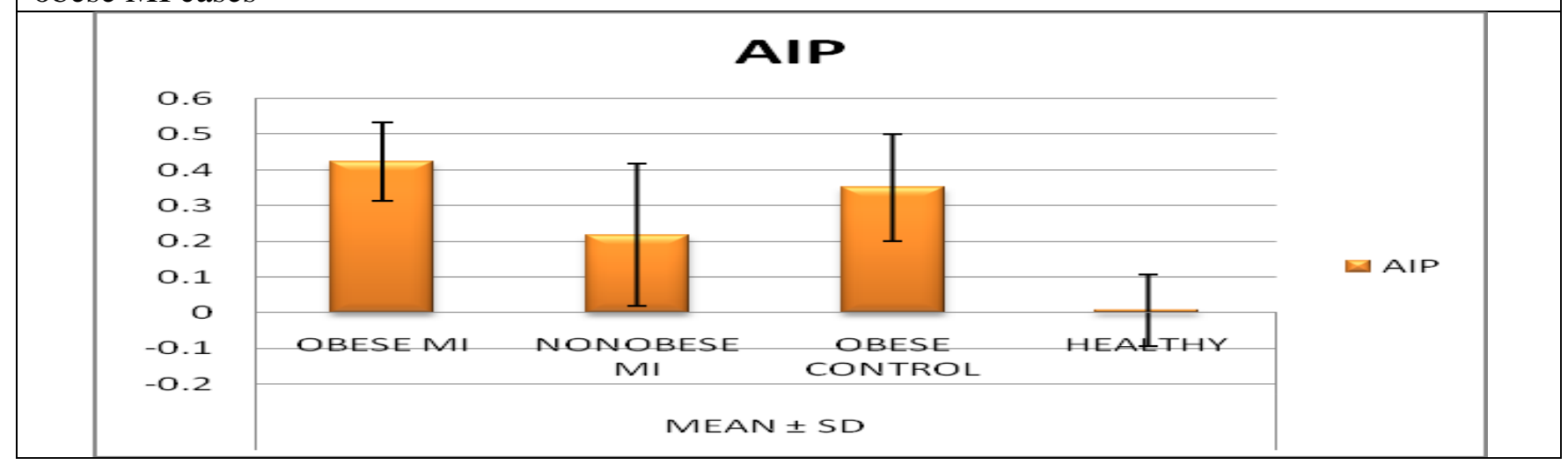


The Mean \pm SD of all the parameters studied in the total cases were significantly different from those of controls. F value was highest for lipid ratios when compared to individual lipids and lipoproteins. Among lipid ratios AIP was found to have higher $\mathrm{F}$ value compared to $\mathrm{CRR}$ and $\mathrm{AC}$. Among the lipids and lipoproteins TAG was found to have higher $\mathrm{F}$ value compared to remaining lipids and lipoproteins. WHR had higher $\mathrm{F}$ value compared to BMI.

\begin{tabular}{|c|c|c|c|c|c|c|c|}
\hline \multicolumn{4}{|c|}{$\begin{array}{l}\text { Table 3. ANOVA multiple Comparison of } \\
\text { significance between healthy controls, obese } \\
\text { controls, non obese MI and obese MI }\end{array}$} & \multicolumn{4}{|c|}{$\begin{array}{l}\text { Table 4. ANOVA multiple Comparison of significance } \\
\text { between obese controls and non obese MI, obese MI; } \\
\text { between non obese MI and obese MI }\end{array}$} \\
\hline \multirow{2}{*}{ Parameter } & \multicolumn{3}{|c|}{ Healthy controls with } & \multirow[t]{2}{*}{ Parameter } & \multicolumn{2}{|c|}{ Obese controls with } & \multirow{2}{*}{$\begin{array}{c}\text { Non obese MI with } \\
\text { Obese MI }\end{array}$} \\
\hline & $\begin{array}{c}\text { Obese } \\
\text { controls }\end{array}$ & $\begin{array}{c}\text { Non obese } \\
\text { MI }\end{array}$ & $\begin{array}{l}\text { Obese } \\
\text { MI }\end{array}$ & & $\begin{array}{c}\text { Non obese } \\
\text { MI }\end{array}$ & $\begin{array}{l}\text { Obese } \\
\text { MI }\end{array}$ & \\
\hline BMI & $<0.001$ & 0.99 & $<0.001$ & BMI & $<0.001$ & 1.000 & $<0.001$ \\
\hline WHR & $<0.001$ & 0.99 & $<0.001$ & WHR & $<0.001$ & 0.619 & \\
\hline TC & $<0.001$ & $<0.001$ & $<0.001$ & TC & 0.534 & 0.997 & 4 \\
\hline $\mathrm{HDL}-\mathrm{C}$ & $<0.001$ & $<0.001$ & $<0.001$ & HDL -C & 0.99 & 0.519 & 46 \\
\hline TAG & $<0.001$ & 0.011 & $<0.001$ & TAG & 0.001 & 0.943 & \\
\hline LDL-C & $<0.001$ & $<0.001$ & $<0.001$ & LDL-C & 0.930 & 505 & \\
\hline CRR & $<0.001$ & $<0.001$ & $<0.001$ & CRR & 0.762 & 024 & \\
\hline $\mathrm{AC}$ & $<0.001$ & $<0.001$ & $<0.001$ & $\mathrm{AC}$ & 0.762 & 0.024 & $<0.001$ \\
\hline AIP & $<0.001$ & $<0.001$ & $<0.001$ & AIP & 0.02 & 0.487 & $<0.001$ \\
\hline \multicolumn{4}{|c|}{$\begin{array}{l}\text { All parameters were significantly increased in obese } \\
\text { controls and obese MI cases compared to healthy } \\
\text { controls except HDL-C which was significantly } \\
\text { decreased. Significant increase was seen in the mean } \\
\text { values of TC, TAG, LDL-C, CRR, AC and AIP in non } \\
\text { obese MI cases compared to healthy controls except } \\
\text { HDL-C which was decreased. The mean values of } \\
\text { WHR and BMI were not significantly different in non } \\
\text { obese MI compared to healthy controls. }\end{array}$} & \multicolumn{4}{|c|}{$\begin{array}{l}\text { In order to assess the maximum sensitivity and specificity exhibited } \\
\text { by various parameters in identifying abnormality the best cut off } \\
\text { values are calculated using ROC analysis. Best cut off values are } \\
\text { established by selecting a point closer to top left hand curve that } \\
\text { provides greatest sum of sensitivity and specificity. The performance } \\
\text { of a diagnostic test can be quantified by calculating Area under curve } \\
\text { (AUC).An ideal test would have a value of } 1 \text {. The cases and controls } \\
\text { were classified as obese and non obese based on BMI. BMI exhibited } \\
100 \% \text { sensitivity and specificity and AUC values of } 1.0 \text { in } \\
\text { discriminating obese and non obese groups. }\end{array}$} \\
\hline
\end{tabular}

Table 5. Best cut off values, sensitivity, specificity in discriminating healthy controls and obese controls

\begin{tabular}{|c|c|c|c|c|}
\hline $\begin{array}{l}\text { Para- } \\
\text { meter }\end{array}$ & $\begin{array}{l}\text { Best Cut } \\
\text { Off Values }\end{array}$ & $\begin{array}{l}\text { Sensi } \\
\text { tivity }\end{array}$ & $\begin{array}{l}\text { Speci - } \\
\text { ficity }\end{array}$ & AUC \\
\hline BMI & 27.65 & $100 \%$ & $100 \%$ & 1.000 \\
\hline WHR & 0.885 & $96.7 \%$ & $96.7 \%$ & 0.957 \\
\hline TC & 179 & $\%$ & $100 \%$ & 0.930 \\
\hline $\mathrm{HDL}-\mathrm{C}$ & 40.5 & $5.7 \%$ & $76.7 \%$ & 0.861 \\
\hline TAG & 153 & $86.7 \%$ & $96.7 \%$ & 0.926 \\
\hline LDL-C & 99.5 & $76.7 \%$ & $93.3 \%$ & 0.887 \\
\hline CRR & 4.3 & $93.3 \%$ & $86.7 \%$ & 0.959 \\
\hline $\mathrm{AC}$ & 2.75 & $93.3 \%$ & $86.7 \%$ & 0.959 \\
\hline AIP & 0.190 & $86.7 \%$ & $96.7 \%$ & 0.932 \\
\hline \multicolumn{5}{|c|}{$\begin{array}{l}\text { AIP and TAG exhibited the highest combined sensitivity and } \\
\text { specificity followed by AC and CRR, TC and LDL-C in } \\
\text { discriminating healthy controls and obese controls. Area } \\
\text { under the curve calculated using ROC analysis showed that } \\
\text { CRR and AC were best discriminatory followed by AIP, TC } \\
\text { TAG ,LDL-C and HDL-C in discriminating healthy controls } \\
\text { and obese controls throughout the range of values studied. }\end{array}$} \\
\hline
\end{tabular}

Table 6. Best cut off values, sensitivity, specificity in discriminating healthy controls and non obese MI

\begin{tabular}{|c|c|c|c|c|}
\hline $\begin{array}{c}\text { Para } \\
\text { meter }\end{array}$ & $\begin{array}{c}\text { Best } \\
\text { Cut Off } \\
\text { Values }\end{array}$ & $\begin{array}{c}\text { Sensi - } \\
\text { tivity }\end{array}$ & $\begin{array}{c}\text { Speci } \\
\text { ficity }\end{array}$ & AUC \\
\hline BMI & 21.85 & 80 & 23.3 & 0.385 \\
\hline WHR & 0.8750 & 16.7 & 90 & 0.402 \\
\hline TC & 150.5 & 83.3 & 70 & 0.860 \\
\hline HDL-C & 40.5 & 86.7 & 77 & 0.811 \\
\hline TAG & 146.5 & 60 & 90 & 0.752 \\
\hline LDL-C & 108 & 66.7 & 100 & 0.884 \\
\hline CRR & 3.8 & 83.3 & 86.7 & 0.907 \\
\hline AC & 2.8 & 83.3 & 86.7 & 0.907 \\
\hline AIP & 0.165 & 73.3 & 96.7 & 0.812 \\
\hline
\end{tabular}

AIP , CRR and AC exhibited highest combined sensitivity and specificity followed by LDL-C , HDL-C , TC and TAG in discriminating non obese MI from healthy controls. Area under the curve calculated using ROC analysis showed that CRR and AC were best discriminatory markers followed by LDL-C , TC, AIP, HDL-C and TAG in discriminating healthy controls and non obese MI cases. 
Table 7. Best cut off values, sensitivity, specificity in discriminating healthy controls and obese MI case group

\begin{tabular}{|c|c|c|c|c|}
\hline Parameter & $\begin{array}{c}\text { Best } \\
\text { Cut Off } \\
\text { Values }\end{array}$ & $\begin{array}{c}\text { Sensit } \\
\text { ivity }\end{array}$ & $\begin{array}{c}\text { Specifi } \\
\text { city }\end{array}$ & AUC \\
\hline BMI & 27.45 & 100 & 100 & 1.000 \\
\hline WHR & 0.89 & 93.3 & 96.7 & 0.956 \\
\hline TC & 176.5 & 80 & 100 & 0.946 \\
\hline HDL-C & 40.5 & 86.7 & 93.3 & 0.929 \\
\hline TAG & 153 & 96.3 & 96.7 & 0.978 \\
\hline LDL-C & 107.5 & 80 & 100 & 0.921 \\
\hline CRR & 4.35 & 86.7 & 96.7 & 0.982 \\
\hline AC & 3.35 & 86.7 & 96.7 & 0.982 \\
\hline AIP & 0.195 & 96.7 & 96.7 & 0.964 \\
\hline
\end{tabular}

AIP was found to have highest combined sensitivity and specificity followed by TAG, CRR, AC, TC, HDL-C and LDL-C in discriminating healthy controls and obese MI case group. Area under the curve calculated using ROC analysis shows CRR and AC best discriminatory followed by TAG, AIP, TC, HDL-C and LDL-C in discriminating healthy controls and obese MI case group.
Table 8. Best cut off values, sensitivity, specificity in discriminating obese controls and non obese MI case group

\begin{tabular}{|c|c|c|c|c|}
\hline $\begin{array}{c}\text { Paramet } \\
\text { er }\end{array}$ & $\begin{array}{c}\text { Best Cut } \\
\text { Off } \\
\text { Values }\end{array}$ & Sensitivity & $\begin{array}{c}\text { Specifi } \\
\text { city }\end{array}$ & AUC \\
\hline BMI & 27.7 & 100 & 100 & 1.000 \\
\hline WHR & 0.89 & 93.3 & 96.7 & 0.991 \\
\hline TC & 258 & 26.7 & 100 & 0.605 \\
\hline HDL-C & 36.5 & 56.7 & 46.7 & 0.496 \\
\hline TAG & 184.5 & 63.3 & 83.3 & 0.782 \\
\hline LDL-C & 171.5 & 26.7 & 93.3 & 0.552 \\
\hline CRR & 5.1 & 76.7 & 46.7 & 0.597 \\
\hline AC & 4.65 & 76.7 & 46.7 & 0.597 \\
\hline AIP & 0.375 & 56.7 & 86.7 & 0.709 \\
\hline
\end{tabular}

TAG was found to have highest combined sensitivity and specificity followed by AIP, CRR, AC, TC, LDL-C and HDL-C in discriminating obese controls and non obese MI case group. Area under the curve calculated using ROC analysis shows TAG best discriminatory followed by AIP, TC, CRR, AC, LDL-C and HDL$\mathrm{C}$ in discriminating obese controls and non obese $\mathrm{MI}$ case group.

Table 9 Best cut off values, sensitivity, specificity in discriminating obese controls and obese MI case group

\begin{tabular}{|c|c|c|c|c|c|c|c|c|c|}
\hline Parameter & $\begin{array}{l}\text { Best Cut Off } \\
\text { Values }\end{array}$ & $\begin{array}{c}\text { Sensitivi } \\
\text { ty }\end{array}$ & $\begin{array}{c}\text { Specifici } \\
\text { ty }\end{array}$ & AUC & $\begin{array}{c}\text { Parame } \\
\text { ter }\end{array}$ & $\begin{array}{c}\text { Best Cut Off } \\
\text { Values }\end{array}$ & $\begin{array}{c}\text { Sensitivi } \\
\text { ty }\end{array}$ & $\begin{array}{c}\text { Specifici } \\
\text { ty }\end{array}$ & AUC \\
\hline BMI & 33.15 & 56.7 & 56.7 & 0.517 & BMI & 27.5 & 100 & 100 & 1.000 \\
\hline WHR & 1.005 & 50 & 70 & 0.588 & WHR & 0.895 & 93.3 & 96.7 & 0.988 \\
\hline $\mathrm{TC}$ & 201.5 & 63.3 & 66.7 & 0.620 & $\mathrm{TC}$ & 257 & 33.3 & 100 & 0.707 \\
\hline HDL-C & 36.5 & 56.7 & 73.3 & 0.66 & HDL-C & 33.5 & 70 & 60 & 0.651 \\
\hline TAG & 182.5 & 80 & 33.3 & 0.536 & TAG & 183 & 80 & 76.7 & 0.837 \\
\hline LDL-C & 115.5 & 76.7 & 46.7 & 0.612 & LDL-C & 155 & 46.7 & 83.3 & 0.676 \\
\hline CRR & 7.35 & 40 & 100 & 0.652 & CRR & 6.95 & 43.3 & 90 & 0.724 \\
\hline $\mathrm{AC}$ & 6.1 & 40 & 100 & 0.652 & $\mathrm{AC}$ & 5.45 & 43.3 & 90 & 0.724 \\
\hline AIP & 0.335 & 83.3 & 40 & 0.625 & AIP & 0.385 & 73.3 & 86.7 & 0.836 \\
\hline \multicolumn{5}{|c|}{$\begin{array}{l}\text { CRR and AC had highest combined sensitivity and } \\
\text { specificity followed by TC, HDL-C, LDL-C, AIP and } \\
\text { TAG in discriminating obese controls and obese MI case } \\
\text { group. Area under the curve calculated using ROC } \\
\text { analysis shows HDL-C best discriminatory followed by } \\
\text { CRR, AC, AIP, TC, LDL-C and TAG in discriminating } \\
\text { obese controls and obese MI case group. }\end{array}$} & \multicolumn{5}{|c|}{$\begin{array}{l}\text { AIP had highest combined sensitivity and specificity followed by } \\
\text { TAG, RR,AC,TC,HDL-C \& LDL-C in discriminating non-obese MI } \\
\text { case group MI \& obese MI case group. Area under the curve } \\
\text { calculated using ROC analysis shows TAG best discriminatory } \\
\text { followed by AIP, CRR, AC, TC, LDL-C and HDL-C in } \\
\text { discriminating non obese MI case group and obese MI case group. In } \\
\text { order to assess the atherogenic risk using cut off values for various } \\
\text { parameters recommended by different authors, individual groups } \\
\text { were stratified. }\end{array}$} \\
\hline
\end{tabular}

Table. 11 Percentage of total patients in different risk groups as classified by Serum Total cholesterol cut off points. $^{16}$

\begin{tabular}{|l|l|c|c|c|c|}
\hline $\begin{array}{l}\text { Total } \\
\text { Cholesterol (mg/dl) }\end{array}$ & $\begin{array}{c}\text { healthy } \\
\text { controls }\end{array}$ & $\begin{array}{c}\text { obese } \\
\text { controls }\end{array}$ & $\begin{array}{c}\text { non obese } \\
\text { MI cases }\end{array}$ & $\begin{array}{c}\text { obese } \\
\text { MI } \\
\text { cases }\end{array}$ \\
\hline Desirable & $<200$ & $100 \%$ & $56.6 \%$ & $63.3 \%$ & $33.3 \%$ \\
\hline Borderline high & $200-239$ & $0 \%$ & $16.6 \%$ & $23.3 \%$ & $26.6 \%$ \\
\hline High & $>240$ & $0 \%$ & $26.6 \%$ & $13.3 \%$ & $40 \%$ \\
\hline
\end{tabular}

None of the healthy controls had cholesterol in the higher risk range while $43.2 \%$ of obese controls, $36.6 \%$ of non obese MI cases and $66.6 \%$ of obese MI cases had cholesterol in higher risk range.
Table 12.Percentage of total patients in different risk groups as classified by Serum HDL cholesterol cut off points. ${ }^{16}$

\begin{tabular}{|c|c|c|c|c|c|}
\hline \multicolumn{2}{|c|}{$\begin{array}{c}\text { HDL } \\
\text { Cholesterol } \\
\text { (mg/dl) }\end{array}$} & \multirow{2}{*}{\begin{tabular}{|c|}
$\begin{array}{c}\text { healthy } \\
\text { controls }\end{array}$ \\
$13.3 \%$ \\
\end{tabular}} & \multirow{2}{*}{$\begin{array}{c}\begin{array}{c}\text { obese } \\
\text { controls }\end{array} \\
76.6 \% \\
\end{array}$} & \multirow{2}{*}{$\begin{array}{c}\begin{array}{c}\text { non obese } \\
\text { MI cases }\end{array} \\
63.3 \% \\
\end{array}$} & \multirow{2}{*}{$\begin{array}{c}\begin{array}{c}\text { obese MI } \\
\text { cases }\end{array} \\
33.3 \% \\
\end{array}$} \\
\hline Low & $\leq 40$ & & & & \\
\hline High & $\geq 60$ & $13.3 \%$ & $0 \%$ & $23.3 \%$ & $26.6 \%$ \\
\hline $\begin{array}{l}\text { range } \\
\text { MI c } \\
\text { highe }\end{array}$ & & $\begin{array}{l}33.3 \% \\
\text { e. }\end{array}$ & ese & ses had & DL- \\
\hline
\end{tabular}


Table 13. Percentage of total patients in different risk groups as classified by Serum Triacylglycerol cut off points. ${ }^{16}$

\begin{tabular}{|c|c|c|c|c|c|c|c|c|c|c|c|}
\hline \multicolumn{6}{|c|}{ cut off points. } & \multicolumn{6}{|l|}{ po1nts. } \\
\hline \multicolumn{2}{|c|}{$\begin{array}{c}\text { Triacylglycerols } \\
(\mathrm{mg} / \mathrm{dl})\end{array}$} & \multirow{2}{*}{$\begin{array}{c}\begin{array}{c}\text { healthy } \\
\text { controls }\end{array} \\
90 \% \\
\end{array}$} & \multirow{2}{*}{$\begin{array}{c}\begin{array}{c}\text { obese } \\
\text { controls }\end{array} \\
13.3 \% \\
\end{array}$} & \multirow{2}{*}{$\begin{array}{c}\begin{array}{c}\text { non } \\
\text { obese } \mathrm{MI} \\
\text { cases }\end{array} \\
50 \% \\
\end{array}$} & \multirow{2}{*}{$\begin{array}{c}\begin{array}{c}\text { obese } \\
\text { MI } \\
\text { cases }\end{array} \\
3.3 \% \\
\end{array}$} & \multicolumn{2}{|c|}{$\begin{array}{c}\text { LDL } \\
\text { Cholesterol } \\
(\mathrm{mg} / \mathrm{dl})\end{array}$} & \multirow{2}{*}{$\begin{array}{c}\begin{array}{c}\text { healthy } \\
\text { controls }\end{array} \\
93.3 \% \\
\end{array}$} & \multirow{2}{*}{\begin{tabular}{c|}
$\begin{array}{c}\text { obese } \\
\text { controls }\end{array}$ \\
$23.3 \%$ \\
\end{tabular}} & \multirow{2}{*}{$\begin{array}{c}\begin{array}{c}\text { non obese } \\
\text { MI cases }\end{array} \\
33.3 \% \\
\end{array}$} & \multirow{2}{*}{$\begin{array}{c}\begin{array}{c}\text { obese MI } \\
\text { cases }\end{array} \\
20 \% \\
\end{array}$} \\
\hline Normal & $<150$ & & & & & Optimal & $<100$ & & & & \\
\hline $\begin{array}{c}\text { Borderline } \\
\text { high }\end{array}$ & $\begin{array}{c}150- \\
199 \\
\end{array}$ & $10 \%$ & $36.6 \%$ & $40 \%$ & $50 \%$ & $\begin{array}{c}\text { Near } \\
\text { optimal }\end{array}$ & $100-129$ & $6.6 \%$ & $36.6 \%$ & $30 \%$ & $23.3 \%$ \\
\hline High & $\begin{array}{c}200- \\
499\end{array}$ & $0 \%$ & $50 \%$ & $10 \%$ & $46.6 \%$ & $\begin{array}{c}\text { Borderline } \\
\text { high }\end{array}$ & $130-159$ & $0 \%$ & $10 \%$ & $23.3 \%$ & $13.3 \%$ \\
\hline \multirow[t]{2}{*}{ Very high } & $>500$ & $0 \%$ & $0 \%$ & $0 \%$ & $0 \%$ & High & $160-189$ & $0 \%$ & $16.6 \%$ & $13.3 \%$ & $13.3 \%$ \\
\hline & & & & & & Very high & $>190$ & $0 \%$ & $13.3 \%$ & $3.3 \%$ & $30 \%$ \\
\hline \multicolumn{6}{|c|}{$\begin{array}{l}10 \% \text { of healthy controls had triacylglycerols in the higher } \\
\text { risk range while } 86.6 \% \text { of obese controls, } 50 \% \text { of non obese } \\
\text { MI cases and } 96.6 \% \text { of obese MI cases had triacylglycerols } \\
\text { in higher risk range. }\end{array}$} & \multicolumn{6}{|c|}{$\begin{array}{l}\text { None of the healthy controls had LDL cholesterol in the higher } \\
\text { risk range while } 39.9 \% \text { of obese controls, } 69.9 \% \text { of non obese } \\
\text { MI cases and } 56.6 \% \text { of obese MI cases had LDL cholesterol in } \\
\text { higher risk range. }\end{array}$} \\
\hline
\end{tabular}

Table 14. Percentage of total patients in different risk groups as classified by Serum LDL cholesterol cut off points. 16

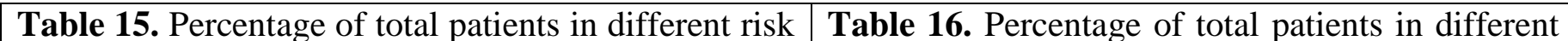
groups as classified by CRR cut off points. ${ }^{17}$

\begin{tabular}{|c|c|c|c|c|c|c|c|c|c|}
\hline \multicolumn{5}{|c|}{ groups as classified by CRR cut off points. } & \multicolumn{5}{|c|}{ risk groups as classified by AIP cut off points. } \\
\hline CRR & $\begin{array}{l}\text { Healthy } \\
\text { Controls }\end{array}$ & $\begin{array}{c}\text { Obese } \\
\text { controls }\end{array}$ & $\begin{array}{c}\text { Non obese MI } \\
\text { cases }\end{array}$ & $\begin{array}{c}\text { Obese MI } \\
\text { cases }\end{array}$ & $\begin{array}{c}\text { AIP } \\
\text { Range }\end{array}$ & $\begin{array}{l}\text { healthy } \\
\text { controls }\end{array}$ & $\begin{array}{c}\text { obese } \\
\text { controls }\end{array}$ & $\begin{array}{l}\text { non obese } \\
\text { MI cases }\end{array}$ & $\begin{array}{c}\text { Obese MI } \\
\text { cases }\end{array}$ \\
\hline $\begin{array}{l}\text { Below avg. risk } \\
\mathrm{M}: 1.0-3.4 \\
\mathrm{~F}: 1.0-3.3\end{array}$ & $73.3 \%$ & $3.3 \%$ & $10 \%$ & $0 \%$ & $\begin{array}{c}\text { Low } \\
\text { risk: }<0.1\end{array}$ & $76.6 \%$ & $10 \%$ & $20 \%$ & $0 \%$ \\
\hline $\begin{array}{l}\text { Avg. risk } \\
M: 3.5-5.0 \\
F: 3.4-4.4\end{array}$ & $26.6 \%$ & $13.3 \%$ & $16.6 \%$ & $13.3 \%$ & $\begin{array}{c}\text { Medium risk: } \\
0.1-0.24\end{array}$ & $3.3 \%$ & $10 \%$ & $6.6 \%$ & $6.6 \%$ \\
\hline $\begin{array}{l}\text { Above avg risk } \\
\mathrm{M}: 5.1-9.6 \\
\mathrm{~F}: 4.5-7.1\end{array}$ & $0 \%$ & $81 \%$ & $73.3 \%$ & $66.6 \%$ & $\begin{array}{l}\text { High risk: } \\
>0.24\end{array}$ & $20 \%$ & $70 \%$ & $73.3 \%$ & $93.3 \%$ \\
\hline $\begin{array}{l}\text { High risk } \\
\mathrm{M}: 9.7-23.4 \\
\mathrm{~F}: 7.2-11.0\end{array}$ & $0 \%$ & $0 \%$ & $0 \%$ & $20 \%$ & \multirow{2}{*}{\multicolumn{5}{|c|}{$\begin{array}{l}23.3 \% \text { of healthy controls had AIP in the higher risk range } \\
\text { while } 80 \% \text { of obese controls, } 79.9 \% \text { of non obese MI cases } \\
\text { and } 99.9 \% \text { of obese MI cases had AIP in higher risk range. }\end{array}$}} \\
\hline \multicolumn{5}{|c|}{$\begin{array}{l}26.6 \% \text { of healthy controls had CRR in the higher risk range } \\
\text { while } 94.3 \% \text { of obese controls, } 89.9 \% \text { of non obese MI cases } \\
\text { and } 99.9 \% \text { of obese MI cases had CRR in higher risk range. }\end{array}$} & & & & & \\
\hline
\end{tabular}

\section{Discussion}

In our study mean values of BMI was significantly increased in obese controls followed by obese MI case group in comparison to the other two groups. The BMI exhibited better discriminatory power than WHR. The ideal discrimination shown by BMI was because it was used to classify a person as obese or nonobese.

WHR is the ratio of the circumference of waist to hip. It is considered as a better predictor of cardiovascular risk than waist circumference and BMI as it is less dependent on body size and height ${ }^{5}$ The mean values of WHR was found to be highest in obese controls followed by obese MI case group in comparison to the other two groups. However in the present study we did not find WHR as a better marker than BMI.

In our study we found mean values of total cholesterol to be significantly higher in obese MI risk groups as classified by AIP cut off points. ${ }^{18}$ cases compared to the remaining groups which is in agreement with other studies where obesity is associated with increased total cholesterol. ${ }^{23}$ The total cholesterol concentrations in obese controls higher compared to healthy controls. Hypercholesterolemia is a well-documented and established risk factor for coronary heart disease (CHD). ${ }^{24}$

Various researches indicate the role of HDL-C as a marker inversely and independently associated with the risk of developing $\mathrm{CHD}^{15}$ Studies have suggested that smaller HDL-C particles have lower free cholesterol content acting as markers associated with the presence of coronary artery disease .

The HDL $-\mathrm{C}$ concentration in obese controls and non obese MI cases are also significantly lower compared to healthy controls. We also observed and non obese MI cases are also significantly of impaired reverse cholesterol transport and 
mean values of $\mathrm{HDL}-\mathrm{C}$ to be lower in obese MI cases and obese controls compared to non obese MI cases. The decreased HDL is due to the impaired lipolysis of triacylglycerol rich lipoproteins (TRL) by decreasing the transfer of apolipoproteins and phospholipids from TRL to the HDL compartment and also by the delayed cleareance of TRLs which facilitates the CETPmediated exchange between cholesterol esters in HDL and triacylglycerols in VLDL. ${ }^{14}$

Studies have indicated that high levels of serum triacylglycerols were not only a stronger risk factor for CHD but were also a better predictor of the severity of atherosclerosis ${ }^{13}$ In our study we found mean values of triacylglycerols to be significantly higher in obese MI case group in comparison to the remaining groups which is in agreement with other studies where obesity is associated with increased triacylglycerols ${ }^{2}$

The increase in triacylglycerols is due to increase in adipocyte mass and the decrease in insulin sensitivity associated with obesity which causes more free fatty acids to be delivered from the adipose tissue to the liver where they are reesterified in hepatocytes to form triacylglycerols, which are packaged into VLDL for secretion into the circulation.

LDL Cholesterol was found to be higher in obese MI cases than remaining groups. These findings are in agreement with other studies ${ }^{23}$ The LDL $-\mathrm{C}$ concentrations were found to be significantly higher in obese controls and non obese MI cases compared to healthy controls. The mean values of LDL-C were higher in obese MI cases and obese controls compared to non obese MI cases.

Mechanistically small dense LDL particles enter the arterial wall more easily and bind to arterial wall proteoglycans more avidly and are highly susceptible to oxidative modification, leading to macrophage uptake all of which may contribute to increased atherogenesis. ${ }^{12}$

Several lipoprotein ratios or atherogenic indices were defined to optimize the predictive capacity of the lipid profile. These ratios include cardiac risk ratio (CRR), atherogenic coefficient (AC) and atherogenic index of plasma (AIP).

The total cholesterol/HDL ratio (Cardiac Risk Ratio) is a superior measure of risk for coronary heart disease compared with either total cholesterol or LDL cholesterol levels. ${ }^{26}$ Various studies have shown CRR to be associated with cardiovascular disease risk. ${ }^{27}$ We found mean values of Cardiac risk ratio to be higher in obese MI cases compared to remaining groups. We observed the mean values of CRR to be significantly higher in obese controls and non obese MI cases compared to healthy controls. Also the mean values of CRR were higher in obese MI cases and obese controls compared to non obese MI cases. Which is in agreement with other studies where obesity is associated with increased $\mathrm{CRR}^{26}$

It has also been demonstrated that Atherogenic coefficient (AC) which is always one unit lower than CRR, can efficiently estimate the ratio of the sum of atherogenic LDL cholesterol and VLDL lipoproteins represented by non-HDL cholesterol to the cardio protective HDL cholesterol which has been supported by several other studies ${ }^{28} \mathrm{We}$ found the mean values of atherogenic coefficient to be higher in obese MI cases compared to remaining groups. This is in agreement with a previous study where atherogenic coefficient is increased in obese compared to controls. ${ }^{23} \mathrm{We}$ observed the mean values of $\mathrm{AC}$ to be significantly higher in obese controls and non obese MI cases compared to healthy controls. The mean values of $\mathrm{AC}$ were found to be higher in obese MI cases and obese controls compared to non obese MI cases in our study.

In our study we found mean value of atherogenic index of plasma (AIP) to be higher in obese MI cases compared to remaining groups which is in agreement with other studies where obesity is associated with increased AIP, ${ }^{25}$ We observed the mean values of AIP to be significantly higher in obese controls and non obese MI cases compared to healthy controls. The mean values of AIP were found to be significantly higher in obese MI cases 
and obese controls compared to non obese MI cases.

F value was found to be highest for lipid ratios when compared to individual lipids and lipoproteins. Among lipid ratios AIP was found to have higher $\mathrm{F}$ value compared to $\mathrm{CRR}$, AC. Among the lipids and lipoproteins TAG was found to have higher $\mathrm{F}$ value compared to remaining lipids and lipoproteins. WHR had higher $\mathrm{F}$ value compared to BMI.

To assess the discriminatory capacity of various markers we used best cut off values as determined by ROC curves. ANOVA multiple comparison of significance showed that CRR and AC were significantly increased in obese MI compared to obese controls and TAG, AIP significantly increased in obese controls compared to non obese MI cases. CRR and AC had highest combined sensitivity and specificity followed by others in discriminating obese controls and obese MI cases. TAG had highest combined sensitivity and specificity followed by AIP, CRR and AC in discriminating obese controls and non obese MI cases. AIP had highest combined sensitivity and specificity in discriminating healthy controls and obese controls, healthy controls and non obese MI cases, healthy controls and obese MI cases, non obese and obese MI cases.

In the present study we also observed that AIP , CRR and TAG predicted the risk in obese MI patients with high sensitivity compared to TC, HDL-C and LDL-C as risk factors. In fact all the obese MI cases are classed as are at higher risk by AIP and CRR. We also observed that in non obese MI patients the risk is predicted more sensitively by CRR and AIP compared to the Lipid and Lipoprotein risk factors tested.

\section{Conclusion}

In the present study Total cholesterol, LDL-C, TAG, and their ratios are significantly increased in obese cases and controls compared to healthy controls, except HDL-C which was decreased. AIP had the highest sensitivity and specificity followed by cardiac risk ratio, atherogenic coefficient and TAG in discriminating healthy controls and remaining groups. Among the lipoprotein ratios log TAG/HDL (AIP) is found to be a better marker in assessing the risk than other ratio and lipoproteins.

We stratified the risk for development of atherosclerosis in the present study using the recommended cutoff values for TC, HDL-C, TAG, LDL-C, CRR and AIP. We observed that lipid ratios are more sensitive predictors of risk for development of atherosclerosis in the study group and that obese subjects are at a higher risk for the development of future atherosclerosis compared to non obese subjects. Among the risk factors studied CRR, TAG and AIP are more sensitive in predicting the future risk of development of atherosclerosis in obese.

As atherogenic lipid profile is associated with atherosclerosis in obesity, the risk identification by using lipid ratios or atherogenic indices were found to be better markers in explaining the pathogenesis of atherosclerosis in these individuals.

\section{Acknowledgements}

Authors thank all the subjects who participated in the study. We express our gratitude to all the faculty and administrators for their kind support and encouragement for this study.

\section{References}

1. A Misra, P Chowbey .Consensus Statement for Diagnosis of Obesity, Abdominal Obesity and the Metabolic Syndrome for Asian Indians and Recommendations for Physical Activity, Medical and Surgical Management JAPI • VOL. 57 • FEBRUARY 2009

2. S. Z. A. Shah, B. R. Devrajani Frequency of dyslipidemia in obese versus non obese in relation to body mass index (BMI), waist hip ratio (WHR) and waist circumference(WC) Pakistan Journal of Science (Vol. 62 No. 1 March, 2010) 
3. F Magkos, BS Mohammed and B Mittendorfer Effect of obesity on the plasma lipoprotein subclass profile in normoglycemic and normolipidemic men and women International Journal of Obesity (2008) 32, 1655-1664

4. Van Gaal LF, Zhang A, Steijaert MM, De Leeuw IH Human obesity: from lipid abnormalities to lipid oxidation. Int J Obes Relat Metab Disord. 1995 Sep;19 Suppl 3:S21-6.

5. RS Gray, RR Fabsitz, etal Relation of generalized and central obesity to cardiovascular risk factors and prevalent coronary heart disease in a sample of AmericanIndians: the Strong Heart StudyInternational Journal of Obesity (2000) 24, 849-860

6. Boban Mathew etal Obesity: Effects on Cardiovascular Disease and its Diagnosis J Am Board Fam Med 2008;21:562- 8.

7. Sonia S. Anand Shofiqul Islam Annika Rosengren ,Risk factors for myocardial infarction in women and men: insights from the INTERHEART study Eur Heart J (2008)doi: 10.1093(8)

8. RS Gray, RR Fabsitz, etal Relation of generalized and central obesity to cardiovascular risk factors and prevalent coronary heart disease in a sample of AmericanIndians: the Strong Heart StudyInternational Journal of Obesity (2000) 24, 849-860

9. Martirosyan, D. M., Miroshnichenko, L. A., Kulokawa, S. N., Pogojeva, A. V. and Zoloedov, V. I. (2007) Amaranth oil application for heart disease and hypertension. Lipids Health Dis. 6:1

10. Chigozie jude ikewuchi and Chidinma catherine ikewuchi, Alteration of Plasma Lipid Profile and Atherogenic Indices of Cholesterol Loaded Rats by Tridax Procumbens Linn: Implications for the Management of Obesity and Cardiovascular Diseases MS/No BKM/2009/043,
(C) 2009 Nigerian Society for Experimental Biology

11. Jesús Millán,1 Xavier Pintó etal Lipoprotein ratios: Physiological significance and clinical usefulness in cardiovascular prevention Vasc Health Risk Manag. 2009; 5: 757-765.

12. Molly c. carr and john d. brunzell .Abdominal Obesity and Dyslipidemia in the Metabolic Syndrome: Importance of Type 2 Diabetes and Familial Combined Hyperlipidemia in Coronary Artery Disease Risk The Journal of Clinical Endocrinology \& Metabolism 89(6):26012607

13. S Johansson, G Bondjers etal Serum lipids and apolipoprotein levels in women with acute myocardial infarction Arterioscler Thromb Vasc Biol 1988, 8:742-749

14. Assmann, G. and Gotto, A. M. Jr. (2004) HDL Cholesterol and Protective Factors in Atherosclerosis. Circulation 109[suppl III]:III-8-III-14

15. Miller GJ, Miller NE. Plasma high-density lipoprotein concentration and development of ischemic heart disease. Lancet. 1975;1:16-20.

16. Evaluation, and Treatment of High Blood Cholesterol in Adults. Executive summary of the third report of the National Cholesterol Education Program (NCEP) expert panel on detection, evaluation, and treatment of high blood cholesterol in adults (Adult Treatment Panel III). JAMA. 2001;285:2486-2497

17. What do my cholesterol levels mean ? American Heart Association/ American stroke

Associationurlhttp://www.heart.org/HEAR TORG/Conditions/Cholesterol/AboutChol esterol/What-Your-Cholesterol-Levels Mean_UCM_305562_Article.jsp

18. Dobiásová M. Atherogenic index of plasma [Log (triglycerides/HDLcholesterol)]: theoretical and practical 
implications. Clin Chem. 2004;50:11131115.

19. Herbert K.,Lipids, In Clinical Chemistry; Theory analysis and Co-relation,Kaplan L.A and Pesce AJ Eds.,1984.p1182-1230.

20. Friedwald W.T.,etal Chem 18,499(1972).

21. Rifai N. Bachorik PS, Albers JJ. Lipids, lipoproteins and apolipoproteins In: Burtis CA, Ashwood ER, editors. Teitz Textbook of clinical chemistry $3^{\text {rd }}$ ed. Philadelphia: W.BSaunders company;1999.p 809-61.

22. Brehm, A., Pfeiler, G., Pacini, G., Vierhapper, H., and Roden, M. (2004) Relationship between Serum Lipoprotein Ratios and Insulin Resistance in Obesity. Clin. Chem. 50: 2316-2322

23. Hanumanthappa nandeesha ,Dr. Zachariah Bobby, etal Atherogenic lipid risk factors in men classifiedas overweight and obese according to the preliminary who guidelines for asiansIndian $\mathrm{J}$ Physiol Pharmacol 2008; 52 (2) : 205-208

24. Ueshima H. Risk assessment chart for death from cardiovascular disease based on a 19-year follow-up study of a Japanese representative population.Circ 2006;70: 1249-1255.

25. Jarosław Derejczyk1, Barbara Kłapcińska, Adolescent Obesity Predicts Cardiovascular Risk Department of Physiological and Medical Sciences, Academy of Physical Education in KatowicePoland.

26. Gianluca etal Comparison of Apolipoprotein (apoB/apoA-I) and Lipoprotein (Total Cholesterol/HDL) Ratio Determinants. Focus on Obesity, Diet and Alcohol Intake.plus.

27. Hong MK, Romm PA, Reagan K, Green CE, Rackley CE. Usefulness of the total cholesterol to high-density lipoprotein cholesterol ratio in predicting angiographic coronary artery disease in women. Am J Cardiol. 1991;68:1646-1650.
28. Stanley H. Hsia, Deyu Pan, ET AL. A Population-Based, Cross-Sectional Comparison of Lipid-Related Indexes for Symptoms of Atherosclerotic Disease. American Journal of Cardiology Volume 98, Issue 8 , Pages 1047-1052, 15 October 2006

29. Fadwa Essiarab, Hassan Taki etal , Usefulness of lipid ratios and atherogenic index of plasma in metabolic syndrome:The Moroccan investigation of cardiovascular diseases Laboratoire de Recherche sur les Lipoprotéines et l'Athérosclérose- Unité de Recherche Casablanca, Morocco 